\title{
Kirchhoff index of a non-complete wheel
}

\author{
Silvia Gago ${ }^{1,2}$ \\ Matemàtiques \\ Universitat Politècnica de Catalunya \\ Barcelona, Spain
}

\begin{abstract}
In this work, we compute analitycally the Kirchhoff index and effective resistances of a weighted non-complete wheel that has been obtained by adding a vertex to a weighted cycle and some edges conveniently chosen. To this purpose we use the group inverse of the combinatorial Laplacian.
\end{abstract}

Keywords: Laplacian matrix, Group inverse, Kirchhoff index, Non-complete wheels.

\section{Introduction and notation}

The invertibility of nonsingular tridiagonal or block tridiagonal matrices has been studied in recent years; see [8] for a review on the subject. Explicit inverses are known in few cases, for instance when the tridiagonal matrix is symmetric with constant diagonals and subject to some restrictions. The techniques used in the mentioned results are mainly based on the theory of orthogonal polynomials. Kirchhoff indexes and effective resistances of networks are very valuable parameters of networks, describing several properties, and

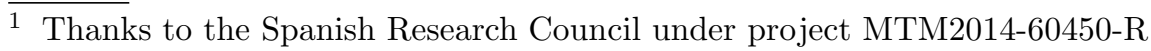

2 Email: \{silvia.gago\}@upc.edu 
therefore have many applications in Electrical networks, Chemistry, etc. (see for instance [2], [6], [9]).

A non-complete wheel is a wheel where the central vertex is connected to a few vertices of the cycle. Many applications of them can be found in Computer Science, as the central vertex is called a hub (see [1]). A non-complete wheel can be seen as a cycle with an added vertex and some new edges and hence, the result of [5] can be applied. In this work, we use the formula for the group inverse to give analytically the effective resistances and the Kirchhoff Index of a non-complete wheel in terms of Chebyshev polynomials.

In the following, the triple $\Gamma=(V, E, c)$ denotes a finite network, that is, a finite graph without loops nor multiple edges, with vertex set $V=\{1, \ldots, n\}$ and edge set $E$, where each edge $e_{i j}=\{i, j\}$ has associated a conductance $c_{i j}>0$. The standard inner product on $\mathbb{R}^{n}$ is denoted by $\langle\cdot, \cdot\rangle$, thus, if $\mathrm{u}$, $\mathrm{v} \in \mathbb{R}^{n}$, then $\langle\mathrm{u}, \mathrm{v}\rangle=\sum_{k=1}^{n} u_{k} v_{k}$. For any $i=1, \ldots, n$ we denote by $\mathrm{e}_{i}$ the $i$-th vector of the standard basis of $\mathbb{R}^{n}$, by $\mathrm{j}$ the all-ones vector of dimension $n$ and by $\mathrm{J}$ the all-ones matrix. Moreover $T_{n}(q), U_{n}(q)$ and $V_{n}(q)$ denote respectively the $n$-th Chebyshev polynomials of first, second and third kind, that is, the Chebyshev polynomials satisfying $T_{0}(q)=U_{0}(q)=V_{0}(q)=1$, $T_{1}(q)=q, U_{1}(q)=2 q$ and $V_{1}(q)=2 q-1$, for any $n \in \mathbb{N}$.

The combinatorial Laplacian or simply the Laplacian of the network $\Gamma$ is the matrix $\mathrm{L}=\left(\mathrm{L}_{i j}\right)$, where $\mathrm{L}_{i i}=\sum_{k=1}^{n} c_{i k}$, and $\mathrm{L}_{i j}=-c_{i j}$ when $i \neq j$. It is well-known that the Laplacian is singular, symmetric and positive semidefinite. Moreover $\mathrm{Lu}=0$ iff $\mathrm{u}$ is proportional to vector $\mathrm{j}$. The group inverse of the Laplacian, $\mathrm{L}^{\#}$, is known as the Green matrix of the network, $\mathrm{G}$.

For every pair of vertices $\{i, j\}$ we define the dipole between them as the vector $\pi_{i j}=\mathrm{e}_{i}-\mathrm{e}_{j}$. Observe that $\pi_{i i}=0$ and $\pi_{i j}=\pi_{j i}$. The effective resistance between two vertices $\{i, j\}$ of a network $\Gamma$, is defined as

$$
R(i, j)=\left\langle\mathrm{G} \pi_{i j}, \pi_{i j}\right\rangle=(\mathrm{G})_{i i}+(\mathrm{G})_{j j}-2(\mathrm{G})_{i j},
$$

and the total resistance of the network or Kirchhoff index (see [2]) is

$$
K(\Gamma)=\frac{1}{2} \sum_{i, j \in V} R(i, j)=n \sum_{i=1}^{n}(\mathrm{G})_{i i}=n \operatorname{trace}(\mathrm{G}) .
$$

For $m>1, d \geq 1$, consider a cycle $C_{n}$ on $n=m d$ vertices with constant conductances $c=c(i, i+1)>0$ for any $i=1, \ldots, n-1$. We define the non-complete wheel $(n, m)-W$, as the network obtained from $C_{n}$ by adding a new vertex $n+1$ to $m$ of the vertices of the cycle placed at the same distance, $d$, with new conductances $a=c(n+1,1+d(i-1))$, for any $i=1, \ldots, m$. 


\section{Effective resistances and Kirchhoff index of non-complete wheels}

In the following section we first give an explicit expression for the effective resistances and Kirchhoff index of the non-complete wheel network $(n, m)-W$, $\mathrm{L}^{\prime}$, in terms of the Laplacian of the base cycle, $\mathrm{L}$.

$$
\mathrm{L}^{\prime}=\left[\begin{array}{cc}
\mathrm{L}+\mathrm{D} & \mathrm{s} \\
\mathbf{s}^{\top} & \alpha
\end{array}\right],
$$

where $\mathrm{D}$ is a diagonal matrix whose non null elements, $a$, are placed at the $(1+d(i-1))$-elements of the diagonal, $i=1, \ldots, m, \mathrm{~s}=-a \sum_{i=1}^{m} \mathrm{e}_{1+d(i-1)}$ and $\alpha=m a$.

Theorem 2.1 The group inverse of $\mathrm{L}^{\prime}$ is

$$
\left(\mathrm{L}^{\prime}\right)^{\#}=\left(\begin{array}{cc}
\mathrm{L}_{11}^{\prime} & \mathrm{L}_{12}^{\prime} \\
\left(\mathrm{L}_{12}^{\prime}\right)^{\top} & \mathrm{L}_{22}^{\prime}
\end{array}\right),
$$

where

$$
\begin{aligned}
\mathrm{L}_{22}^{\prime} & =\frac{12 c d n+a n\left(d^{2}-1\right)}{12 a c(n+1)^{2}} \\
\mathrm{~L}_{12}^{\prime} & =-\frac{d}{a(n+1)^{2}}\left(\frac{a}{12 c d}\left(d^{2}-1\right)+n+2\right) \mathbf{n} \otimes \mathbf{j}_{m},
\end{aligned}
$$

with

$$
(\mathrm{n})_{i}=-\frac{a}{12 c n}(n(6+d)+5 m+6 m i(i-d-2)), \text { for any } i=1, \ldots, d,
$$

and where $\mathrm{L}_{11}^{\prime}$ is the block Toeplitz matrix

$$
\mathrm{L}_{11}^{\prime}=\left[\begin{array}{cccc}
\mathrm{N}_{1} & \mathrm{~N}_{2} & \ldots & \mathrm{N}_{m} \\
\mathrm{~N}_{m} & \mathrm{~N}_{1} & \ddots & \vdots \\
\vdots & \ddots & \ddots & \mathrm{N}_{2} \\
\mathrm{~N}_{2} & \ldots & \mathrm{N}_{m} & \mathrm{~N}_{1}
\end{array}\right]
$$


such that any submatrix $\mathrm{N}_{k}, k=1, \ldots, m$, has entries

$$
\begin{aligned}
\left(\mathrm{N}_{k}\right)_{i h+1} & =\frac{1}{2 c n}(-|i-1-k(d-1)-h|(n-|i-1-k(d-1)-h|) \\
& +\frac{1}{T_{m}(q)-1}\left[n(h-i+1)\left(V_{k-1}(q)-V_{m-k}(q)\right)\right. \\
& -\left(\frac{a n}{c}(i-1)(h-d)-n d\right)\left(U_{k-2}(q)+U_{m-k}(q)\right) \\
& -2 c d \\
& \left.+\left(V_{m-1}(q)-1\right)-d^{2} U_{m-1}(q)\right]+d h+n h-n d \\
& +\frac{1}{n+1}\left(\frac{(n-1)\left(d^{2}-1\right)}{6}-(i-2-d) i-h^{2}+d h-d-1\right) \\
& \left.+\frac{a\left(d^{2}-1\right)+12 c d}{6 a(n+1)^{2}}\right),
\end{aligned}
$$

with $i=1, \ldots, d h=0, \ldots, d-1$ and $q=\frac{a d}{2 c}+1$.

The proof of this result can be found in [7]. Once computed the group inverse, the effective resistances between two vertices and the Kirchhoff index of the new network are easily obtained.

Proposition 2.2 Given two vertices of $\Gamma^{\prime}$, the effective resistances of the network between them are:

a) if $i, j \neq n+1, i=\left(k_{1}-1\right) d+h_{1}, j=\left(k_{2}-1\right) d+h_{2}$ and $k^{\prime}=k_{2}-k_{1}+1$, where $1 \leq k_{1} \leq k_{2} \leq n$ and $h_{1}, h_{2}=0, \ldots, d-1$, with $h_{1}<h_{2}$ when $k_{1}=k_{2}$, 
then

$$
\begin{aligned}
R(i, j)= & \frac{-1}{T_{m}(q)-1}\left[\frac{\left(h_{2}-h_{1}\right)}{c}\left(V_{k^{\prime}-1}(q)-V_{m-k^{\prime}}(q)\right)-\left(\frac{a}{c^{2}} h_{1}\left(h_{2}-d\right)-\frac{d}{c}\right)\right. \\
& \left.\left(U_{k^{\prime}-2}(q)+U_{m-k^{\prime}}(q)\right)+\left(\frac{a}{2 c^{2}}\left(h_{1}^{2}+h_{2}^{2}-\left(h_{1}+h_{2}\right) d\right)-\frac{d}{c}\right) U_{m-1}(q)\right] \\
+ & \frac{1}{c n}\left(\left|h_{1}-h_{2}-k^{\prime}(d-1)\right|\left(n-\left|h_{1}-h_{2}-k^{\prime}(d-1)\right|\right)\right. \\
- & \left(2 k^{\prime} d+2 h_{2}\right) h_{1}-k^{\prime} d\left(n-2 h_{2}\right)+d^{2}\left(k^{\prime}-1\right)^{2}+h_{1}^{2}+h_{2}^{2} \\
+ & \left.\left(3 h_{1}-2 h_{2}\right) d+n\left(h_{1}-h_{2}+d\right)\right)-\frac{(n-1)^{2}\left(d^{2}-1\right)}{6 c(n+1)^{2}}
\end{aligned}
$$

b) if $i=\left(k_{1}-1\right) d+h_{1} \neq n+1$ and $j=n+1$, then

$$
\begin{aligned}
R(i, n+1)= & -\frac{1}{T_{m}(q)-1}\left[\frac{d}{a n}\left(V_{m-1}(q)-1\right)+\frac{1}{2 c n}\left(\frac{a n}{c}\left(h_{1}^{2}-h_{1} d\right)-n d+d^{2}\right)\right. \\
& \left.\cdot U_{m-1}(q)\right]+\frac{h_{1}^{2}-h_{1} d}{c(n+1)^{2}}+\frac{12 c d\left(n^{2}+1\right)+a n^{2}\left(d^{2}-1\right)}{12 a c n(n+1)^{2}} \\
& \frac{\left(6 h_{1}^{2}-6 h_{1} d-1\right)}{6 c(n+1)^{2}}\left(\frac{a\left(d^{2}-1\right)}{12 c d}+n+2\right),
\end{aligned}
$$

with $q=\frac{a d}{2 c}+1$. Moreover, the Kirchoff index of $\Gamma^{\prime}$ is

$$
\begin{gathered}
K\left(\Gamma^{\prime}\right)=-\frac{1}{2 c} \frac{n+1}{T_{m}(q)-1}\left[\left(\frac{a n}{6 c}-d n+d^{2}\right) U_{m-1}(q)+\left(\frac{2 c d}{a}+\frac{d n}{3}\right)\right. \\
\left.\left(V_{m-1}(q)-1\right)\right]+\frac{d}{a}+\frac{d n(n+1)}{6 c}-\frac{(d-1)^{2} n}{12 c} .
\end{gathered}
$$

Finally, observe that if we consider the case where $d=1, n=m$, we obtain a Wheel, and in thus the obtained Kirchhoff index coincides with the result of $[2]$. 
Corollary 2.3 The Kirchhoff index of a Wheel on $n+1$ vertices is

$$
\begin{aligned}
K\left(\Gamma^{\prime}\right) & =-\frac{1}{2 c} \frac{n+1}{T_{n}(q)-1}\left[\left(\frac{a n}{6 c}-n+1\right) U_{n-1}(q)+\left(\frac{2 c}{a}+\frac{n}{3}\right)\left(V_{n-1}(q)-1\right)\right] \\
& +\frac{1}{a}+\frac{n(n+1)}{6 c} .
\end{aligned}
$$

\section{References}

[1] Golumbic M.C., Stern M., Levy A., Morgenstern G., "Graph-Theoretic Concepts in Computer Science: 38th International Workshop", WG 2012, Jerusalem, Israel, June 26-28, 2012, Revised Selected Papers. Springer, 2012 Edition.

[2] Bendito E., Carmona A., Encinas A.M., Gesto J.M., A Formula for the Kirchhoff Index, Int. J. Quantum Chem., 108 (2008), 1200-1206.

[3] Carmona A., Encinas A.M., Mitjana M., Discrete elliptic operators and their Green operators, Linear Algebra Appl., 442 (2014), 115-134.

[4] Carmona A., Encinas A.M., Gago S., Jiménez M.J., Mitjana M., The inverse of some circulant matrices, Appl. Math. Comput., 270 (2015), 785-793.

[5] Carmona A., Encinas A.M., Gago S., Mitjana M., Green operators of networks with a new vertex, Linear Algebra Appl., 491 (2016), 419-433.

[6] Cinkir Z., Deletion and contraction identities for the resistance values and the Kirchhoff index, Int. J. Quantum Chem., 111 (2011), 4030-4041.

[7] Gago S., The Group Inverse of extended Symmetric and Periodic Jacobi Matrices, submitted.

[8] Meurant G., A review on the inverse of symmetric tridiagonal and block tridiagonal matrices, SIAM J. Matrix Anal. Appl., 13 (1992), 707-728.

[9] Wang W., Yang D., Luo Y., The Laplacian polynomial and Kirchhoff index of graphs derived from regular graphs, Discrete Appl. Math., 161 (2013), 30633071. 